\title{
Efficacy and safety of levosimendan in Chinese elderly patients with Takotsubo syndrome
}

\author{
Yi Guo ${ }^{1 \#}$, Chaofei Zhou" ${ }^{1 \#}$, Xia Yang ${ }^{2}$ \\ ${ }^{1}$ Department of Cardiology, Hainan Branch of Chinese People's Liberation Army General Hospital, Sanya 572013, China; ${ }^{2}$ Department of \\ Cardiology, Chinese People's Liberation Army General Hospital, Beijing 100853, China \\ Contributions: (I) Conception and design: All authors; (II) Administrative support: None; (III) Provision of study materials or patients: All authors; \\ (IV) Collection and assembly of data: All authors; (V) Data analysis and interpretation: All authors; (VI) Manuscript writing: All authors; (VII) Final \\ approval of manuscript: All authors. \\ "These authors contributed equally to this work. \\ Correspondence to: Xia Yang. Department of Cardiology, Chinese People's Liberation Army General Hospital, Fuxing Road 28, Haidian District, \\ Beijing 100853, China. Email: yxhn1980@126.com.
}

\begin{abstract}
Background: Takotsubo syndrome has a low global incidence, but it is observed more and more in clinical practice. Only case reports and series have been published, while prospective studies are still necessary. This study aimed to explore the efficacy and safety of levosimendan in elderly with Takotsubo syndrome.

Methods: This study followed a prospective, randomized and double-blinded design. All 200 consecutive patients (>65 years) with Takotsubo syndrome were randomly assigned into a levosimendan group $(n=100)$ and a control group $(n=100)$. The control group underwent regular treatment, and the levosimendan group was additionally administrated with levosimendan.

Results: Age of all patients was 71 [66-85] years, and there were 138 females (69\%). Baseline characteristics between the two groups had no significant difference before treatment ( $>>0.05$ for all). In the levosimendan group on the 30 and 180 days after treatment, left ventricular ejection fraction was significantly higher, and New York Heart Association class and N-terminal pro-brain natriuretic peptide levels were significantly lower, than those in the control group $(\mathrm{P}<0.05$ for all). Systolic blood pressure, diastolic blood pressure, heart rate and serum creatinine levels had no significant difference between the two groups $(\mathrm{P}>0.05$ for all).

Conclusions: Levosimendan showed reliable efficacy and safety in Chinese elderly patients with Takotsubo syndrome, supporting the idea that levosimendan has the potential to be an essential drug applied for patients with Takotsubo syndrome.
\end{abstract}

Keywords: Chinese elderly; levosimendan; Takotsubo syndrome

Submitted Jun 01, 2018. Accepted for publication Sep 21, 2018.

doi: $10.21037 /$ atm.2018.10.15

View this article at: http://dx.doi.org/10.21037/atm.2018.10.15

\section{Introduction}

Although Takotsubo syndrome has a low global incidence, it is observed more and more in current clinical practice $(1,2)$. Levosimendan is a new type of $\mathrm{Ca}^{2+}$ sensitizer that can improve cardiac function without severely affecting stable hemodynamics and renal function $(3,4)$. However, to date there has hardly been any prospective study to observe the application of levosimendan in patients with Takotsubo syndrome, and there are only controversial results based on the clinical experience (1). As advised by the current position statement from the Heart Failure Association of European Society of Cardiology, the published literature on the Takotsubo syndrome only consists of case reports and series, while prospective studies to probe the efficacy and safety of levosimendan in patients with Takotsubo syndrome are still necessary (1).

China, the largest developing country in the world, has 
become an aging society, and there are increased morbidity and mortality rates in the elderly $(5,6)$. Clinical data on the levosimendan application in Chinese elderly patients with Takotsubo syndrome remain very rare, and it is essential to analyze the efficacy and safety of levosimendan in these patients. The current study aimed to explore the efficacy and safety of levosimendan in patients with Takotsubo syndrome.

\section{Methods}

\section{Study participants}

The current study followed a prospective, randomized and double-blinded design. A total of 200 consecutive patients over the age of 65 who had Takotsubo syndrome were admitted to the Department of Cardiology in our hospital from January 2013 to December 2016. Takotsubo syndrome was diagnosed according to the consensus from chief physicians of the Cardiology Department and in line with the diagnostic criteria of the Mayo Clinic and the position statement from the Heart Failure Association of the European Society of Cardiology. Inclusion criteria were in the following: (I) transient regional ventricular wall motion abnormalities; (II) absence of culprit atherosclerotic coronary artery disease or other pathological conditions, such as hypertrophic cardiomyopathy and viral myocarditis, to explain the pattern of ventricular dysfunction; (III) new electrocardiography abnormalities; (IV) significantly elevated serum N-terminal pro-brain natriuretic peptide (NTproBNP) levels; (V) positive but relatively small elevation in cardiac troponin T; (VI) recovery of ventricular function at follow-up (6 months) $(1,7,8)$. Patients were excluded based on the following criteria: (I) sensitivity or intolerance to levosimendan and other formulation ingredients; (II) severe ventricular filling and outflow obstruction; (III) electrolyte disturbance (serum potassium $<3.5$ or $>5.5 \mathrm{mmol} / \mathrm{L}$ ); (IV) severe hepatic and renal impairment; (V) severe hypotension and tachycardia including ventricular tachycardia or ventricular fibrillation (9).

\section{Study procedures}

Patients were randomly assigned into a levosimendan group $(n=100)$ and a control group $(n=100)$ using random numbers in a randomized block design. Random numbers were generated by Statistic Package for Social Science (SPSS) version 17.0 software (SPSS Inc., Chicago, USA).
After diagnosed with Takotsubo syndrome and assigned into the two groups, the control group underwent regular treatment, and the levosimendan group was administrated with levosimendan (Qilu Pharmaceutical, Jinan, China; Specifications: $5 \mathrm{~mL}, 12.5 \mathrm{mg}$ ) at continuously intravenous infusion of $0.1 \mu \mathrm{g} / \mathrm{kg} / \mathrm{min}$ without loading dose for 24 hours in addition to regular treatment $(1,10)$. Drug use was a standardized process. All patients received coronary angiography and left ventriculography. They all had continuous strict electrocardiographic and hemodynamic monitoring. Left ventricular ejection fraction (LVEF) was measured using Simpson's method through standard echocardiography. The blood sample was drawn from all patients and then analyzed in the Department of Biochemistry. Serum NT-proBNP levels were measured by NT-proBNP Flex Reagent Cartridge (PBNP/LPBN) produced by Siemens Healthcare Diagnostics Inc (Deerfield, USA) on the Dimension RxL Max (Siemens Healthcare Global, Erlangen, Germany). Serum creatinine levels were measured by enzymatic assay (Roche Diagnostics GmbH) on the Hitachi 7600 autoanalyzer (Hitachi, Tokyo, Japan). All patients were followed up on the 30 and 180 days after treatment $(1,11)$. All patients were not re-hospitalized after discharge from hospital. They received follow-up and reexamination in the Outpatient Department in our hospital.

\section{Statistical analyses}

Continuous variables with normal distribution were reported using mean and standard deviation, and the difference between the two groups was compared using Student's $t$-test. Continuous variables with abnormal distribution were reported using median and interquartile range, and the difference between the two groups was compared using Mann-Whitney $U$ test. Categorical variables were reported with number and percentage, and the difference between the two groups was compared with Chi-square test. All analyses were carried out by SPSS version 17.0 software (SPSS Inc., Chicago, USA), and $P$ value $<0.05$ was accepted as statistically significant.

\section{Results}

Age of all patients was 71 [66-85] years, and there were 138 females (69\%). Baseline characteristics between the two groups had no significant difference before treatment ( $\mathrm{P}>0.05$ for all; Table 1). Comparison between the two groups on the 30 and 180 days after treatment is shown in 
Table 1 Baseline characteristics of elderly patients with Takotsubo syndrome in the levosimendan and control group

\begin{tabular}{|c|c|c|c|}
\hline Characteristics & Levosimendan group $(n=100)$ & Control group $(n=100)$ & $\mathrm{P}$ \\
\hline Females, n (\%) & $71(71.0)$ & $67(67.0)$ & 0.541 \\
\hline Hypertension, n (\%) & $21(21.0)$ & $17(17.0)$ & 0.471 \\
\hline Diabetes mellitus, $\mathrm{n}(\%)$ & $11(11.0)$ & $13(13.0)$ & 0.663 \\
\hline $\mathrm{DBP}(\mathrm{mmHg})$ & 70 [64-72] & 68 [64-74] & 0.573 \\
\hline Heart rate (time/minute) & 93 [89-103] & 97 [90-102] & 0.232 \\
\hline NYHA class, n [\%] & & & 1.000 \\
\hline IV & $100[100]$ & $100[100]$ & \\
\hline Serum creatinine $(\mu \mathrm{mol} / \mathrm{L})$ & $79.0(71.0-98.5)$ & $85.6(75.9-92.5)$ & 0.353 \\
\hline ACEI/ARB, n (\%) & $29(29.0)$ & $23(23.0)$ & 0.333 \\
\hline Beta-blockers, n (\%) & 0 & 0 & 1.000 \\
\hline Diuretics, n (\%) & $83(83.0)$ & $87(87.0)$ & 0.428 \\
\hline
\end{tabular}

ACEI/ARB, angiotensin converting enzyme inhibitor/angiotension receptor blocker; DBP, diastolic blood pressure; NT-proBNP, N-terminal pro-brain natriuretic peptide; NYHA, New York Heart Association; LVEF, left ventricular ejection fraction; SBP, systolic blood pressure.

Table 2. In the levosimendan group, LVEF was significantly higher, and New York Heart Association (NYHA) class and serum NT-proBNP levels were significantly lower, than those in the control group $(\mathrm{P}<0.05$ for all). Systolic blood pressure, diastolic blood pressure, heart rate and serum creatinine levels had no significant difference between the two groups ( $\mathrm{P}>0.05$ for all). Other kinds of side effects were not found in the two groups. Compared with the control group, mortality was significantly lower in the levosimendan group $(\mathrm{P}<0.05)$. All patients died of circulatory failure.

\section{Discussion}

Takotsubo syndrome has a low global incidence (1). The treatment of Takotsubo syndrome in elderly patients should be taken into special consideration (1). As advised by the current position statement from the Heart Failure Association of European Society of Cardiology, inotropes, including dobutamine and dopamine, should be forbidden in patients with Takotsubo syndrome, as further activation of catecholamine receptors or other molecular pathways might aggravate their condition $(12,13)$. Levosimendan is one of the limited options that is left, but there have been rare case reports and series to observe the application of levosimendan in patients with Takotsubo syndrome, and prospective studies to probe the efficacy and safety of levosimendan are still necessary, especially in Chinese elderly patients (1). The current study found that levosimendan improved cardiac function and reduced mortality in elderly patients, which suggests that levosimendan is effective in treating Takotsubo syndrome in Chinese elderly patients. Meanwhile, these elderly patients in the current study exhibited no distinction of blood pressure, heart rate and renal function with the treatment of levosimendan or not, which suggests that levosimendan is safe in treating Takotsubo syndrome in Chinese elderly patients. Therefore, levosimendan is very appropriate for Chinese elderly patients with Takotsubo syndrome.

Levosimendan is a new type of $\mathrm{Ca}^{2+}$ sensitizer that can improve cardiac function without severely affecting stable hemodynamics and renal function $(3,4)$. Levosimendan has provided beneficial cardioprotective and hemodynamic effects in cardiac surgery field and patients with septic shock $(4,14,15)$. However, there has hardly been any prospective studies analyzing the application of levosimendan in patients with Takotsubo syndrome. The current study 
Table 2 Efficacy and safety of elderly patients with Takotsubo syndrome in the levosimendan and control group

\begin{tabular}{|c|c|c|c|}
\hline Characteristics & Levosimendan group $(n=100)$ & Control group $(n=100)$ & $\mathrm{P}$ \\
\hline \multicolumn{4}{|l|}{30 days } \\
\hline NYHA class, n (\%) & & & 0.041 \\
\hline ॥ & $68(68.7)$ & $48(52.2)$ & \\
\hline III & $29(29.3)$ & $38(41.3)$ & \\
\hline LVEF [\%] & $47[44-55]$ & $47[41-51]$ & 0.035 \\
\hline NT-proBNP (pg/mL) & $759.3(545.5-1,116.9)$ & $897.6(660.8-1,118.2)$ & 0.044 \\
\hline Serum creatinine $(\mu \mathrm{mol} / \mathrm{L})$ & 83.6 (73.6-103.0) & $88.3(71.1-99.0)$ & 0.596 \\
\hline $\mathrm{SBP}(\mathrm{mmHg})$ & $124[121-132]$ & $125[114-136]$ & 0.852 \\
\hline Mortality, n (\%) & $1(1.0)$ & $8(8.0)$ & 0.041 \\
\hline \multicolumn{4}{|l|}{180 days } \\
\hline NYHA class, n (\%) & & & 0.042 \\
\hline I & $68(68.7)$ & $50(54.3)$ & \\
\hline ॥ & $31(31.3)$ & $42(45.7)$ & \\
\hline LVEF [\%] & 49 [47-57] & 49 [47-53] & 0.040 \\
\hline NT-proBNP (pg/mL) & $476.7(245.5-664.6)$ & $542.5(360.8-748.7)$ & 0.032 \\
\hline Serum creatinine $(\mu \mathrm{mol} / \mathrm{L})$ & $83.0(73.7-101.0)$ & $86.3(69.1-97.7)$ & 0.669 \\
\hline
\end{tabular}

DBP, diastolic blood pressure; NT-proBNP, N-terminal pro-brain natriuretic peptide; NYHA, New York Heart Association; LVEF, left ventricular ejection fraction; SBP, systolic blood pressure.

proved favorable efficacy and safety of levosimendan in Chinese elderly patients with Takotsubo syndrome. It is not surprising that levosimendan has beneficial effects on the elderly patients with cardiac troponin $\mathrm{C}$ insensitive to $\mathrm{Ca}^{2+}$. Levosimendan can be combined with cardiac troponin $\mathrm{C}$ for the greater sensitivity to $\mathrm{Ca}^{2+}$, leading to myocardial contraction even at the same or lower $\mathrm{Ca}^{2+}$ concentration $(16,17)$.

\section{Conclusions}

The current study demonstrated that levosimendan showed reliable efficacy and safety in Chinese elderly patients with
Takotsubo syndrome, supporting the idea that levosimendan has the potential to be an essential drug applied for patients with Takotsubo syndrome.

\section{Acknowledgements}

We are grateful to all study participants for their participation in the study.

\section{Footnote}

Conflicts of Interest: The authors have no conflicts of interest to declare. 
Ethical Statement: The study protocol has been approved by Ethics Committee of Chinese People's Liberation Army General Hospital (Beijing, China; ID: 301hn11201601) and it conforms to the Helsinki Declaration. Each participant provided written informed consent to be included in the study.

\section{References}

1. Lyon AR, Bossone E, Schneider B, et al. Current state of knowledge on Takotsubo syndrome: a position statement from the task force on Takotsubo syndrome of the Heart Failure Association of the European Society of Cardiology. Eur J Heart Fail 2016;18:8-27.

2. Mann DL. The emerging role of small non-coding RNAs in the failing heart: big hopes for small molecules. Cardiovasc Drugs Ther 2011; 25:149.

3. Nieminen MS, Fruhwald S, Heunks LM, et al. Levosimendan: current data, clinical use and future development. Heart Lung Vessel 2013;5:227-45.

4. Toller W, Algotsson L, Guarracino F, et al. Perioperative use of levosimendan: best practice in operative settings. J Cardiothorac Vasc Anesth 2013;27:361-6.

5. Lu J, Wang H, Pan Y. Analysis of determinants of population longevity at county level in China. Popul Econ 2004;146:13-8.

6. Jugdutt BI. Aging and heart failure: changing demographics and implications for therapy in the elderly. Heart Fail Rev 2010;15:401-5.

7. Prasad A, Lerman A, Rihal CS. Apical ballooning syndrome (Tako-Tsubo or stress cardiomyopathy): a mimic of acute myocardial infarction. Am Heart J 2008;155:408-17.

8. Ponikowski P, Voors AA, Anker SD, et al. 2016 ESC Guidelines for the diagnosis and treatment of acute and chronic heart failure: The Task Force for the diagnosis

Cite this article as: Guo Y, Zhou C, Yang X. Efficacy and safety of levosimendan in Chinese elderly patients with Takotsubo syndrome. Ann Transl Med 2018;6(22):438. doi: 10.21037/atm.2018.10.15 and treatment of acute and chronic heart failure of the European Society of Cardiology (ESC). Developed with the special contribution of the Heart Failure Association (HFA) of the ESC. Eur Heart J 2016;37:2129-200.

9. Rossi S. Australian Medicines Handbook. 2006.

10. Santoro F, Ieva R, Ferraretti A, et al. Safety and feasibility of levosimendan administration in takotsubo cardiomyopathy: a case series. Cardiovasc Ther 2013;31:e133-7.

11. Follath F, Cleland JG, Just H, et al. Efficacy and safety of intravenous levosimendan compared with dobutamine in severe low output heart failure (the LIDO study): a randomised double-blind trial. Lancet 2002;360:196-202.

12. Redmond M, Knapp C, Salim M, Shanbhag S, Jaumdally R. Use of vasopressors in Takotsubo cardiomyopathy: a cautionary tale. Br J Anaesth 2013;110:487-8.

13. Shao Y, Redfors B, Stahlman M, et al. A mouse model reveals an important role for catecholamine-induced lipotoxicity in the pathogenesis of stress-induced cardiomyopathy. Eur J Heart Fail 2013;15:9-22.

14. Altenberger J, Parissis JT, Ulmer H, Poelzl G. Rationale and design of the multicentre randomized trial investigating the efficacy and safety of pulsed infusions of levosimendan in outpatients with advanced heart failure (LevoRep study). Eur J Heart Fail 2010;12:186-92.

15. Salmenpera M, Eriksson H. Levosimendan in perioperative and critical care patients. Curr Opin Anaesthesiol 2009;22:496-501.

16. Zhang D, Yao Y, Qian J, et al. Levosimendan improves clinical outcomes of refractory heart failure in elderly Chinese patients. Med Sci Monit 2015;21:2439-45.

17. Parissis JT, Rafouli-Stergiou P, Paraskevaidis I, et al. Levosimendan: from basic science to clinical practice. Heart Fail Rev 2009;14:265-75. 